\title{
Effect of focus on verbal working memory: Critical role of the focus word in reading
}

\author{
MARIKO OSAKA, YUKIKO NISHIZAKI, and MIE KOMORI \\ Osaka University of Foreign Studies, Osaka, Japan \\ and \\ NAOYUKI OSAKA \\ Kyoto University, Kyoto, Japan
}

\begin{abstract}
The effect of focus on working memory was investigated with the reading span test (RST). In two experiments, the span scores and the number of intrusion errors were compared between the focused RST the and the nonfocused RST. Focus word was defined as the most important word for comprehending a sentence. For the focused RST, the target word to be maintained was the focus word for the sentence. In the nonfocused RST, however, the target word was not the focus word for the sentence. The results of both experiments showed that RST span scores were higher for the focused RST than for the nonfocused RST, and intrusion errors were found to increase for the nonfocused RST. In Experiment 2, the effect of focus was compared between high-span and low-span subjects. An effect of sentence length was also investigated. The result showed that low-span subjects were more affected than were high-span subjects by whether the word to be remembered was the focus word. The effect of sentence length was not confirmed. These findings suggest that the low-span subjects had deficits in their ability to establish and/or inhibit mental focus when faced with conflict situations in reading.
\end{abstract}

Working memory refers to the immediate brain processes involved in the simultaneous storage and processing of information and plays an important role in complex cognition, such as language comprehension, learning, and reasoning (Baddeley, 1986; Just \& Carpenter, 1992).

Working memory plays a particularly critical role in comprehension processes in text reading. For example, incoming information is decoded perceptually, reorganized, and integrated with the contextual interpretation, and the constituent products of each of these processes are stored for a short period (Daneman \& Carpenter, 1980; Kintsch \& Van Dijk, 1978). In this way, working memory is important in storing the intermediate and final products of successive data, allowing the reader to integrate the contents and place the text words into context.

There are individual differences in working memory capacity, and these differences account for some aspects of language comprehension (Just \& Carpenter, 1992). To investigate the involvement of working memory in reading comprehension, Daneman and Carpenter (1980) developed the reading span test (RST). During the RST, subjects read a few sentences aloud while maintaining the last word of

The work was supported in part by Grants 14310041 from the Ministry of Education and Science of Japan to the first author and 12301005 to the last author. Thanks are due Jay Rueckl for comments and suggestions regarding the manuscript. Correspondence concerning this article should be addressed to M. Osaka, Department of Psychology, Osaka University of Foreign Studies, Aomadani-Higashi, Mino, Osaka 562-8558, Japan (e-mail: osaka@post01.osaka-gaidai.ac.jp). each sentence in memory. Daneman and Carpenter's (1980) resource-sharing model of working memory contends that the mental resources available for both processes (reading a sentence and storing the last word of each sentence) are finite and that subjects must allocate portions of these resources during the RST. Thus, the RST measures both processing and storage functions during reading. Daneman and Carpenter (1980) found a significant correlation between reading comprehension scores and RST span scores. Subjects with a large working memory capacity (highspan subjects for the RST) were successful in interpreting the meaning of an ambiguous word when it was distant from the portion of the text necessary to clarify its meaning. However, subjects with a small working memory capacity (low-span subjects for the RST) had difficulty in maintaining target words, owing to insufficient working memory capacity while reading. Although a significant correlation between performance on the RST and reading comprehension has been found by many studies (Baddeley, Logie, Nimmo-Smith, \& Brereton, 1985; Daneman \& Carpenter, 1980; Masson \& Miller, 1983), there was little or no significant correlation has been found between reading comprehension and a short-term memory task (Daneman \& Carpenter, 1980; Perfetti \& Goldman, 1976).

\section{What Does the RST Measure?}

Several hypotheses have been proposed to explain which of the processes required for the RST are involved in sentence comprehension. Daneman and Carpenter (1983) have suggested that semantic processing of sentence com- 
prehension is attributable to differences in capacity: Highspan subjects devote fewer resources to the semantic processing of sentences; therefore, they still have sufficient resources to maintain the words. It was also suggested not only that high-span subjects were quicker to process linguistic materials, but also that they made greater use of various strategies (Carpenter \& Just, 1989). Moreover, Daneman and Merikle (1996) performed a meta-analysis of studies in which working memory span tasks have been used and showed that passive storage measures correlated less with reading skills than did working memory span measures.

However, La Pointe and Engle (1990) investigated the effect of word length with a simple word span task and with the RST, specifically looking at the relationship between these tasks and reading comprehension. Word length had an effect on both tasks, and both tasks correlated with reading comprehension. On the basis of these results, they proposed another explanation of processing with the RST - namely, that RST did measure short-term memory capacity, rather than working memory function.

Other researchers have suggested that an inhibitory mechanism is important. Regarding inhibition mechanisms, Gernsbacher (1990) suggested that poor readers lack a good inhibitory mechanism and that this deficit is implicated in comprehension difficulty. In the experiment by Gernsbacher and Faust (1991), subjects were told to focus on either a picture or a word in the context display and to ignore the other. They were then asked to indicate whether a word in a test display was related to the word in the context display. There were differences between skilled and less skilled readers in terms of ignoring an irrelevant stimulus. The results suggested that the suppression mechanism of less skilled readers is less efficient in suppressing the nonfocused ignored information than is that of more skilled readers.

Similar suggestions have been made regarding those who perform poorly on the RST (Conway \& Engle, 1994; De Beni, Palladino, Pazzaglia, \& Cornoldi, 1998; Engle, Conway, Tuholski, \& Shisler, 1995; May, Hasher, \& Kane, 1999). These studies have shown that low working memory task performers have a deficit in inhibiting information that is irrelevant for the task.

Towse, Hitch, and Hutton (1998) proposed an alternative interpretation to the resource-sharing model. They reported that sentence processing and word storage did not compete for working memory resources in the RST. In their experiment, the sentence lengths of the first and last sentences were changed. When the first sentence was short and the last sentence was long, the subjects had to hold the last word of the first sentence in memory for a longer period. However, when the first sentence was long and the last sentence was short, the subjects held the word for a shorter period. The results showed that RST scores were higher for the latter condition than for the former condition. They concluded that subjects performing the RST task utilized a task-switching strategy in which they alternated reading the sentence and holding the last word (Towse, Hitch, \& Hutton, 2000).
In these studies, however, little attention has been paid to the semantic processing instrumental in the comprehension of sentences. With the RST, it is a critical issue whether or not the target word is a word that is important for comprehending the sentence. When a target word to be maintained coincides with the focus word of the sentence, word maintenance is likely easier, and RST performance is enhanced. However, when a target word does not coincide with the focus word of the sentence, the subjects will have poor performance on the RST. Thus, when investigating the role of working memory in reading, it is necessary to address the role played by the focus word of the sentence.

\section{What Is Focus?}

Traditionally, the focus of the sentence pertains to information that is new and unrecoverable from preceding discourse, and focus structure is divided into two parts: a focused part containing new information and a remaining part containing previously given information (Jackendoff, 1972). The focus of a sentence is commonly indicated by assigning stress to a focus word (Cutler, 1976). In a phonememonitoring task, Cutler showed that the stressed focus word shortened reaction time. However, stress is not the only method used for focusing on a word in a sentence. Linguistic theory asserts that a preceding question can restrict the focus of a proper response sentence (Chomsky, 1971). In Cutler and Fodor (1979), focus was manipulated by having a question precede the sentence. As a result, when the preceding question had focused on the earlier part of the sentence, reaction time in the phoneme monitoring task to the earlier target in a sentence was faster, and when the focus was on the later part of the sentence, reaction time to the later target was faster. On the basis of these results, it has even been suggested that focus accelerates sentence comprehension processes (Cutler \& Fodor, 1979).

Psychologically, focus structure is one of the main factors controlling the listener's attention in language comprehension, so that he or she pays more attention to the processing of focused sentence parts than to processing of nonfocused parts (Hornby, 1974). Blutner and Sommer (1988) showed that sentence focus had an effect on the lexical processing of ambiguous words during language comprehension. In their experiment, the focus-identifying task of Cutler and Fodor (1979) was adapted for testing the processing of an ambiguous word. Blutner and Sommer showed that both meanings of an ambiguous word were activated if the ambiguous word formed part of the semantic focus; however, the activation of both meanings was not so strong if the ambiguous word was outside the focus. They suggested that focus plays an important role in sentence comprehension and, furthermore, that focus of a sentence is one of the main factors in the controlling of attention in language comprehension.

With a text-reading task, Birch and Garnsey (1995) also have investigated the effect of focus on memory for words in sentences and have found that focusing a word enhances memory. They proposed that focus in the sentence facilitates the process of the integration of information in sentences 
and is critical for creating a coherent understanding. Carpenter and Just (1977) also have suggested that subjects initially direct their attention to the focus of a sentence and that focused constituents are then easily comprehended during reading.

\section{Hypothesis}

On the basis of these results, it can be supposed that focus in the sentence plays an important role in text reading with comprehension. That is, readers direct their attention to the word that is critical for text integration. When they encode the meaning of each word in a sentence, they have to decide whether the word is relevant or irrelevant to the core information needed to integrate the sentence. Whenever a subject is reading a sentence, he or she is most likely to search for the most important word-that is, the focus word that provides an advantage for integration of the sentence.

The same process could be applicable to the RST, just as for sentence comprehension. With an RST, when a target word to be maintained coincides with the focus word of the sentence, word maintenance is likely easier, and RST performance is enhanced. However, when a target word does not coincide with the focus word of the sentence, subjects are faced with a conflict between the target word and the focus word and, as a result, will have poor performance on the RST. In addition, they will be more apt to falsely report the focus word instead of the target word, leading to an increase in the number of intrusion errors on the RST.

In the present study, the effect of focus on a target word during performance of an RST was investigated. Adopting the definition from Halliday (1967) and Birch and Garnsey (1995), we defined the focus word as "the most important to comprehend a sentence"- that is, the word with a core meaning necessary to integrate the sentence.

In order to identify the focus word of each sentence, focus words were selected with student evaluations in a preliminary investigation. The students were requested to identify which word of the sentence was the most important and critical to the understanding of the sentence. As in Birch and Garnsey (1995), focus words were adopted when more than $70 \%$ of the students selected the word as the focus word of the sentence.

In two experiments, we investigated the focus effect of the target word on the RST scores and on intrusion errors, using Japanese sentences. In both experiments, two kinds of RSTs were used. One was a focused RST in which the target word to be maintained was a focus word of the sentence. The other was a nonfocused RST in which the target word was another word (nonfocus word) in the sentence. In both kinds of RSTs, the target word was underlined and occupied various positions, except for the first and the last positions in a sentence.

As for the target position in the sentences in the Japanese version of the RST, the target word occupied various positions in the sentence and was the underlined word in each sentence (Osaka \& Osaka, 1992, 1994). In studies of RST in English, the target word has been the last word of each sentence (Daneman \& Carpenter, 1980). This differ- ence in target position in our Japanese version was required because the last word of a sentence in Japanese is usually a verb. Moreover, as a feature of Japanese syntactic mechanisms, the last word of a Japanese sentence is rarely a focus word (Kuno, 1980), whereas in English, the last word of the sentence easily becomes the end focus word (Bolinger, 1986). Comparing Japanese RSTs and English RSTs, we found a significantly high correlation between the Japanese RST span scores and the English RST span scores ( $r=$ $.84, p<.001$; Osaka \& Osaka, 1992). Moreover, a significant correlation was found between the Japanese RST span scores and the scores of reading comprehension test scores $(r=.50, p<.05$; Osaka \& Osaka, 1994).

In Experiment 1, the goal of the subjects was to read a set of sentences and to remember one word from each sentence. The word to be remembered was either the focus of the sentence (focused RST) or another word in the sentence (nonfocused RST). We measured the span scores and the intrusion errors for both RST types. Intrusion errors were nontarget words belonging to the sentence that were incorrectly recalled.

In Experiment 2, the effect of focus on RST span scores and intrusion errors was compared between high working memory span subjects and low working memory span subjects. Moreover, an effect of sentence length was investigated to determine whether sentence length interfered with the focus effect. Therefore, we compared the effect of focus across short and long sentences in the RST.

\section{EXPERIMENT 1}

\section{Method}

Identification of the focus word. One hundred forty sentences in Japanese were selected, each of which contained 25-30 characters. In Japanese, sentence length is suggested to be properly measured in mora, a phoneme unit (Koizumi, 1978). Therefore, we also measured sentence lengths in mora. The lengths of the stimulus sentences were 30-40 moras. All of the sentences contained more than two nouns. Each of the two nouns ranged from 2 to 4 characters and was 2-5 moras in length. These nouns were controlled for their word frequency (frequency value ranged from 100 to 300), using standard Japanese word frequency values (Amano \& Kondo, 1999).

In a preliminary investigation, 100 undergraduate students were asked to choose a focus word from each of 140 sentences. After reading each sentence, they were requested to evaluate which word was the most important and was critical to understanding the sentence. Focus words were adopted when more than $70 \%$ of the students selected one of the two nouns we had already chosen. As a result of this procedure, 70 sentences and their focus noun words were selected.

Focused RST and nonfocused RST. From these sentences, two kinds of sentences were made, corresponding to focused RST and nonfocused RST types. In both kinds of RSTs, the same 70 sentences were used. In the focused RST, the target word to be remembered was the focus noun of the sentence. In the nonfocused RST, however, the target word was not the focus word but another nonfocus noun. The evaluation rate for the nonfocus nouns by the students in the preliminary investigation of ours was below $18 \%$.

The target words were controlled for word frequency (198.4 in the focused RST and 201.4 in the nonfocused RST). To equalize the concrete values of the target words, concreteness values were also measured for the target words, using a 7-point rating scale ( 7 being most concrete). The concrete values of the target words were 5.29 in the focused RST and 5.37 in the nonfocused RST. 
Table 1 shows sample sentences from the focused RST and the nonfocused RST, with an English translation of each sample sentence. In the sentence "The child dropped food on his jacket and made stains," the word "stains "was chosen as the focus word. Therefore, in the focused RST, "stains" was selected as the target word, whereas in the nonfocused RST version, the noun "jacket" was selected as the target word.

In each trial of both RSTs, there was no semantic relationship among the sentences, and there was no phonetic or semantic similarity among the target words. Also, the position of the target word in the sentence was controlled in both RSTs.

Subjects. Sixty Japanese undergraduate students performed the task; half of the subjects performed the focused RST task, and the other half performed the nonfocused RST task. All of the subjects performed all the trials on each RST task.

Procedure. We followed the procedure used by Daneman and Carpenter (1983). The subjects were asked to read sets of sentences aloud and to remember the underlined word in each sentence. After the last sentence of each set was read, the subjects were requested to recall the target word of each sentence orally. The number of sentences in a set was increased from two to five. There were five trials of each set size. The order of recalling the target word was unconditional, except that the last target was to be reported first in order to avoid recency effects. The span value was evaluated as the highest level at which a subject could correctly answer three of five sets, and if the subject correctly recalled only two of five sets at a particular level, the subject was given a score of 0.5 for that level. RST performance was measured as the span score.

\section{Results}

RST span scores and intrusion error. The mean span scores for 30 subjects for the two kinds of RSTs were 3.67 $(S D=0.95)$ for the focused RST and $3.00(S D=0.89)$ for the nonfocused RST. ${ }^{1}$ The mean span score was significantly higher for the focused RST than for the nonfocused $\operatorname{RST}[t(58)=2.76, p<.01]$.

We also counted the number of intrusion errors. Intrusion errors were the number of nontarget words belonging to the set of sentences that were incorrectly recalled. The mean number of total intrusion errors during the focused RST and the nonfocused RST was $1.03(S D=1.02)$ and 3.77 $(S D=2.47)$, respectively. A significant difference was also found for the intrusions $[t(58)=3.31, p<.01]$ between the focused RST and the nonfocused RST.

In the nonfocused RST, intrusion errors were divided into two types. Focus intrusion occurred when the subject reported the focus word instead of the target word, and nonfocus intrusion occurred when the subject reported a word other than the focus word. The mean number of focus intrusions and nonfocus intrusions was 2.37 ( $S D=$ $1.83)$ and $1.40(S D=1.33)$, respectively. It was found that the number of focus intrusions was significantly larger than that of nonfocus intrusions $[t(29)=2.34, p<.01]$.

\section{Discussion}

In Experiment 1, span scores were significantly higher for the focused RST than for the nonfocused RST. Thus, we confirmed the focus effect on memory for the target word in the RST.

One explanation for the differences in results between the focused RST and the nonfocused RST is that a failure to facilitate comprehension processes during the nonfo- cused RST is involved. In the focused RST task, it was easier for the subjects to maintain the target word, because the target word was in accord with the focus word of the sentence. In the nonfocused RST, however, recall of the target word was not facilitated by comprehension processes, because the target word was not in accord with the focus of the sentence.

It would be expected that the recall of focused and nonfocused targets would not differ if the subjects alternated between reading the sentence and storing the target word independently, as Towse et al. (1998) have suggested. However, our results did indeed show that the subjects performed better in the focused RST task than in the nonfocused RST task. The results of Experiment 1 cannot ultimately confirm whether or not the subjects adapted a strategy of task switching, because the enhancement of the focus word, making it more easily recalled than the nonfocus word, as Birch and Garnsey (1995) have found, is facilitated by intrinsic comprehension processes most likely independent of task strategy. Nevertheless, Towse, et al. (1998) confirmed the effects of their task-switching strategy by citing decreased span scores with longer retention delays. We further investigated this problem in Experiment 2, using sentences with two different lengths.

Another explanation for the differences in results between the focused RST and the nonfocused RST is that there was an interaction between the focus and the target words in the nonfocused RST task. In the nonfocused RST task, the target word was not in accord with the focus word of the sentence; it was more difficult for the subject to isolate the target word for processing, because of interference from the focus word, which had already been allotted attentional resources. Thus, poorer performance was observed for the nonfocused RST.

In the present results, the number of intrusion errors was significantly higher when the target word was a nonfocus word than when it was a focus word. Furthermore, most of the intrusion errors on the nonfocused RST task were errors in which the subjects reported the focus word instead of the nonfocus target word. The predominance of focus intrusion errors with the nonfocused RST also suggests that an interaction occurred between the focus word and

Table 1

Sample Sentences for the Focused RST and the Nonfocused RST Focused RST:

その子供は洋服に食べ物を落としてしみをつけた。

Target Word: しみ focus word: しみ

The child dropped food on his jacket and made stains.

Target word: stains focus word: stains

Non-Focused RST:

その子供は洋服に食べ物を落としてしみをつけた。

Target Word: 洋服 focus word: しみ

The child dropped food on his jacket and made stains.

Target word: jacket focus word: stains 
the target word - that is, the subjects had difficulty inhibiting the focus word.

These results support other studies that have indicated that poor comprehenders make significantly more intrusion errors than do good comprehenders (Gernsbacher, 1990; Gernsbacher \& Faust, 1991). In this experiment, however, the subjects made significantly more intrusion errors in the nonfocused RST condition than in the focused RST condition. With respect to the task itself, the focus word and its significance for comprehension of the sentence should have been irrelevant in the nonfocused RST task. However, the subjects had difficulty inhibiting the focus word. The theory of Gernsbacher, which implicates a lack of inhibition of irrelevant stimuli, would predict equal intrusion errors for the focused RST and the nonfocused RST tasks. We investigated this issue further by comparing high working memory capacity subjects and low working memory capacity subjects in Experiment 2.

\section{EXPERIMENT 2}

In Experiment 2, the focus effect was compared between high working memory subjects and low working memory subjects. The focus effect was also investigated across sentence length.

\section{Method}

Subjects. To identify high versus low working memory capacity individuals, 60 subjects were tested with standard versions of the Japanese RST ${ }^{2}$ (Osaka \& Osaka, 1992, 1994) in a preliminary experiment. The mean RST span score of the 60 subjects was $3.10(S D=$ 0.90). Incorporating the criterion applied by Just and Carpenter (1992), we selected two groups of subjects: those who had span scores ranging from 4.0 to 5.0 and those who had span scores ranging from 2.0 to 2.5 , respectively named high-span subjects and low-span subjects. Twenty-three high-span subjects and 23 low-span subjects were selected. The mean span score was $4.3(S D=0.74)$ for highspan subjects and $2.1(S D=0.72)$ for low-span subjects. None of these subjects had participated in Experiment 1.

Short and long focused RST and nonfocused RST. One hundred fifty short sentences and 150 long sentences were selected. The short sentences ranged from 25 to 30 characters (30-40 moras) in length, whereas the long sentences ranged from 35 to 40 characters (40-50 moras) in length. All the sentences contained more than two nouns. The word length of each of the two nouns ranged from 2 to 4 characters (2-5 moras). The nouns were controlled for word frequency, as in Experiment 1. Concreteness values of the nouns were also measured, using a 7-point rating scale.

One hundred undergraduate students were asked to choose a focus word from each of the sentences. As a result, there were 84 short focused RST sentences and 84 long focused RST sentences created. With these sentences, corresponding long and short nonfocused RST sentences were created by identifying a target other than the focus word.

In this way, four RST conditions were constructed. There were 84 short focused RST sentences, 84 long focused RST sentences, 84 short nonfocused RST sentences, and 84 long nonfocused RST sentences. The mean sentence lengths, in mora, were $33.7(S D=2.42)$ for the short focused RST, $33.5(S D=2.32)$ for the short nonfocused RST, $44.7(S D=3.51)$ for the long focused RST, and $44.6(S D=3.48)$ for the long nonfocused RST. The mean position of the target word in each sentence was $13.98(S D=1.22)$ for the short focused RST, $13.21(S D=1.18)$ for the short nonfocused RST, $19.79(S D=2.02)$ for the long focused RST, and 20.37 ( $S D=2.30$ ) for the long non- focused RST. The word frequency values were 215.1 (short focused RST), 207.5 (short nonfocused RST), 202.1 (long focused RST), and 223.0 (long nonfocused RST). The concrete values were 5.0 (short focused RST), 5.7 (short nonfocused RST), 5.3 (long focused RST), and 5.5 (long nonfocused RST).

In the nonfocused RST, half of the target words preceded the focus words, and the other half of the target words followed the focus words. As in Experiment 1, in each trial of the RST task, there was no semantic relationship among sentences and no phonetic or semantic similarity among target words.

A methodological objective in this experiment was to have each subject tested in each condition. Since the focused and the nonfocused RSTs contained identical sentences, each RST condition was halved into sentence lists of 42 each-in essence, creating two equal versions of the experiment, with different sentences in each condition. Half of the subjects in each high- and low-span group performed the task with one version, and the other half of the subjects performed the task with the other version.

Procedure. The procedure was the same as that in Experiment 1, except that each condition in which there were two to five sentences had three trials. Because each subject performed four kinds of RSTs, we limited the trial number for each RST, in order to avoid their having to read an excessive number of sentences aurally. The presentation order of the conditions was counterbalanced across subjects. The span value was evaluated as the level at which a subject correctly answered two of three sets. If the subject correctly reported only one of the three sets at a particular level, the subject was given a score of 0.5 at that level.

\section{Results}

Individual difference in RST span score. RST span scores were analyzed for 23 high-span subjects and 23 lowspan subjects. For high-span subjects, the mean focused RST span score was $4.37(S D=0.74)$ in the short-sentence condition and $4.63(S D=0.64)$ in the long-sentence condition. The mean nonfocused RST span score was 4.33 $(S D=0.78)$ in the short-sentence condition and 4.33 $(S D=0.76)$ in the long-sentence condition. For low-span subjects, the mean focused RST span score was 3.39 $(S D=0.80)$ in the short-sentence condition and 3.52 $(S D=1.00)$ in the long-sentence condition. The mean nonfocused RST was $2.85(S D=0.90)$ in the short-sentence condition and $2.85(S D=0.75)$ in the long condition. ${ }^{3} \mathrm{~A}$ three-way analysis of variance (ANOVA), with group (high-span subjects and low-span subjects), focus (focused and nonfocused), and sentence length (short and long) as factors, revealed that there was a significant main effect of focus $\left[F(1,44)=20.789, M S_{\mathrm{e}}=0.480, p<.0001\right]$. There was also a significant main effect of group $[F(1,44)=$ $50.239, p<.0001]$. There was a significant interaction between focus and subject group $[F(1,44)=6.416, p<$ .05]. However, there were neither a main effect of RST sentence length nor interactions between sentence length and the other variables. Since a three-way interaction was not significant, and because sentence length had no main effects or interactions, we will present all data and analyses collapsed over conditions of sentence length. Figure 1 shows the mean span scores for both the focused RST and the nonfocused RST for both subject groups. Further analysis showed that the span score was higher for the focused RST $(M=3.46, S D=0.90)$ than for the nonfocused RST $(M=2.85, S D=0.82$; Fisher, $p<.0001)$ for low-span subjects. For high-span subjects, however, there was no 


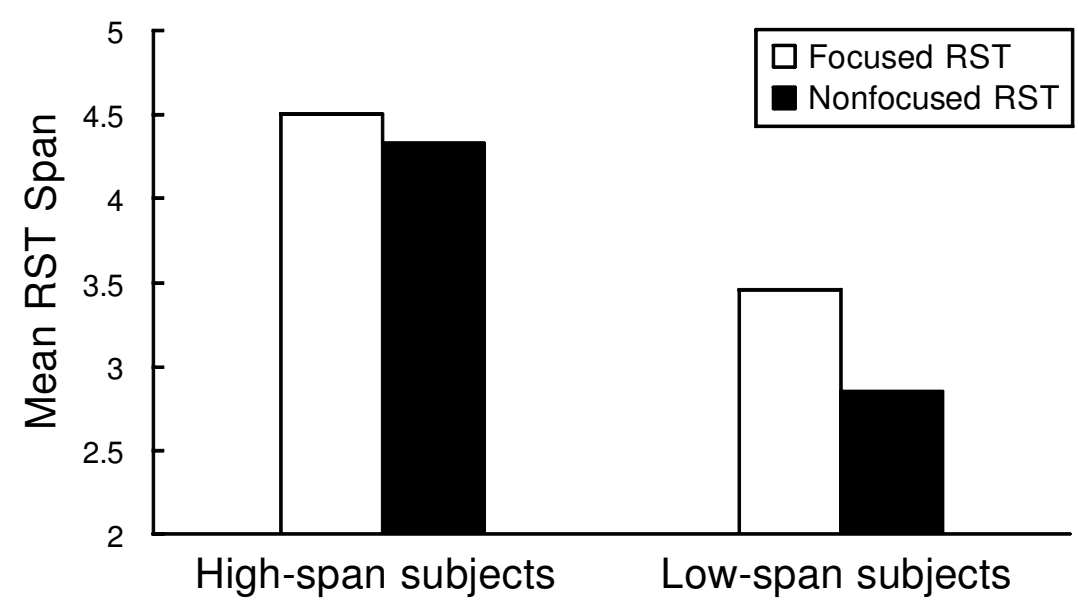

Figure 1. The mean span scores for both the focused RST and the nonfocused RST for high-span subject and low-span subject groups.

difference in the span score between the focused RST and the nonfocused RST.

Group differences in intrusion errors. We computed the total number of intrusion errors for each subject group. The mean number of intrusion errors for high-span subjects for the focused RST was $0.74(S D=0.81)$ in the shortsentence condition and $0.44(S D=0.78)$ in the long-sentence condition. For the nonfocused RST, the mean number of intrusion errors was $1.91(S D=1.70)$ in the short-sentence condition and $1.44(S D=1.33)$ in the long-sentence condition. For low-span subjects, the mean number of intrusion errors for the focused RST was $1.04(S D=1.17)$ in the short-sentence condition and $0.87(S D=0.86)$ in the long-sentence condition. For the nonfocused RST, the error number rose to $3.44(S D=2.50)$ in the short-sentence condition and $3.44(S D=2.6)$ in the long-sentence condition. A three-way ANOVA (group, focus, and sentence length) revealed that there were significant main effects of focus $\left[F(1,44)=50.419, M S_{\mathrm{e}}=1.628, p<.0001\right]$ and group $[F(1,44)=9.735, p<.01]$. There was a significant interaction between focus and subject group $[F(1,44)=$ $7.678, p<.01]$; however, there was not a main effect of sentence length or an interaction between sentence length and the other variables. Since a three-way interaction was not significant, and because sentence length had no main effects or interactions, we will present all data and analyses collapsed over condition of sentence length. Figure 2 shows the intrusion errors for both the focused RST and the nonfocused RST for both subject groups. The effect of focus on intrusion error was confirmed in both subject groups. The number of intrusion errors was significantly greater for the nonfocused RST than for the focused RST for high-span subjects $[F(1,22)=14.47, p<.001]$ and low-span subjects $[F(1,22)=36.04, p<.0001]$. The effect of focus was stronger for the low-span subjects than for the high-span subjects. Moreover, a significant group

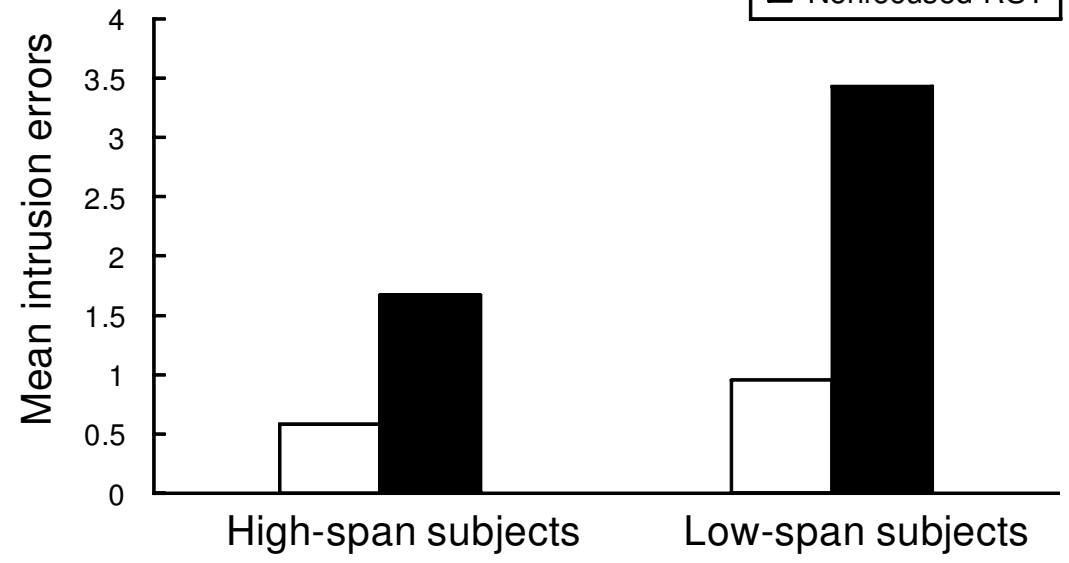

Figure 2. The mean numbers of intrusion errors for both the focused RST and the nonfocused RST for high-span subject and low-span subject groups. 


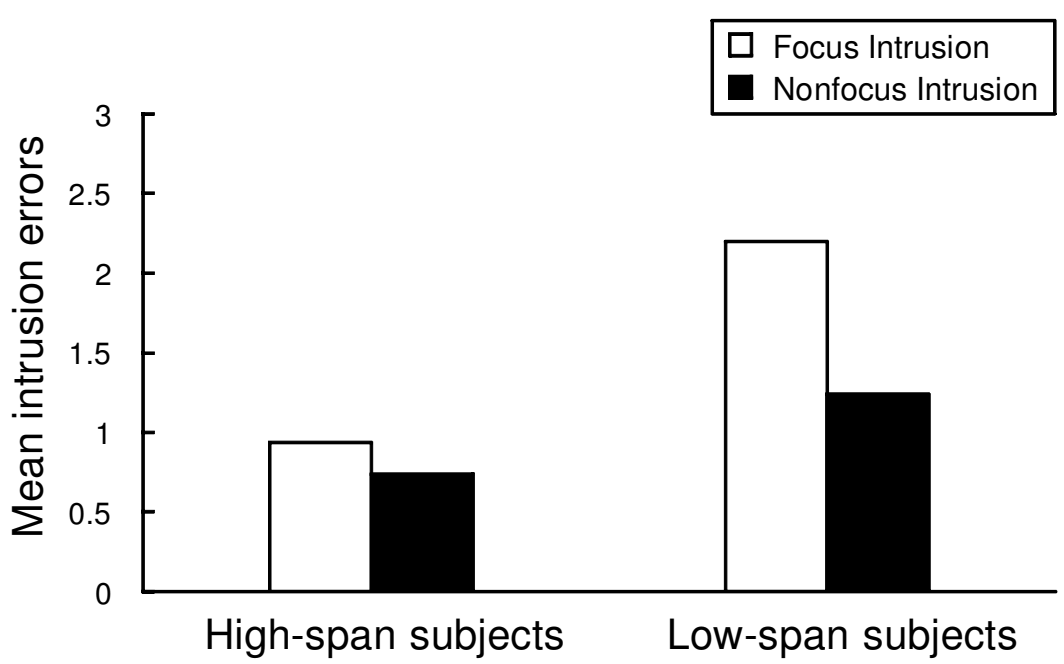

Figure 3. The mean numbers of focus intrusion and nonfocus intrusion errors for both subject groups for nonfocused RST.

difference was confirmed both for the focused RST (Fisher, $p<.01$ ) and for the nonfocused RST (Fisher, $p<$ $.0001)$.

Focus intrusion versus nonfocus intrusion. For the nonfocused RST only, focus intrusion error and nonfocus intrusion error was compared between high-span subjects and low-span subjects. For high-span subjects, the mean number of focus intrusion errors was $1.21(S D=1.50)$ in the short-sentence condition and $0.65(S D=0.98)$ in the longsentence condition, and the mean number of nonfocus intrusion errors was $0.70(S D=0.87)$ in the short-sentence condition and $0.78(S D=0.90)$ in the long-sentence condition. For low-span subjects, the mean number of focus intrusion errors was $2.22(S D=1.90)$ in the short-sentence condition and $2.17(S D=1.72)$ in the long-sentence condition, and the mean number of nonfocus intrusion errors was $1.22(S D=1.12)$ in the short-sentence condition and $1.26(S D=1.48)$ in the long-sentence condition. A threeway ANOVA was performed, with intrusion type (focus intrusion and nonfocus intrusion), subject groups (high and low span subjects), and sentence length (short and long) as factors. The analysis revealed that there were significant main effects of intrusion type $[F(1,44)=9.831$, $\left.M S_{\mathrm{e}}=1.245, p<.01\right]$ and group $[F(1,44)=9.778, p<$ $.01]$. There was a significant interaction between intrusion type and subject group $[F(1,44)=4.287, p<.05]$. However, there was no main effect of sentence length or any interaction among sentence length, group, and intrusion type. A three-way interaction was not significant. Figure 3 shows the numbers of focus intrusion errors and nonfocus intrusion errors for both subject groups. Further analysis showed that for high-span subjects, there was no significant difference between the types of intrusion errors. For low-span subjects, however, a significant difference between focus intrusion $(M=2.20, S D=1.80)$ and nonfocus intrusion $(M=1.24, S D=1.20)$ was found (Fisher, $p<.01)$.

\section{Discussion}

Focus and sentence length. In Experiment 2, performance was better when the targets consisted of focus words than when they consisted of nonfocus words, with no effect of sentence length or interaction between the focus word and sentence length. These results suggest that the focus effect was independent of sentence length.

In the present experiment, there were substantial differences between short and long sentences, more than 10 moras. In the four-sentence condition, for example, when the subjects had to read aurally three additional sentences after reading the first, the maintenance period of holding the first target word before report was an additional 30 moras longer in the long-sentence condition than in the short-sentence condition. Despite this, there was no difference in span scores between long and short sentences. If sentence length mattered, the span score would be expected to decrease, owing to the longer maintenance time for the target word. Whenever the sentence became longer, additional reading time was required. If the subjects for the present results had alternated between reading and storing the target word, as Towse et al. (1998, 2000) suggested, they would have shown worse performance on the RST with longer maintenance. The present results, however, showed that maintenance time did not have a strong effect on span scores, which suggests that the subjects did not use a task-switching strategy.

There is one explanation for the lack of sentence length effects in the present study. Identification of the focus word was high for all of the sentences used in both the focused RST and the nonfocused RST; the percentages were higher than $70 \%$. Therefore, the mental representation that the readers made while reading the sentence, via focus word facilitation, was rather stable and was hardly disturbed by sentence length. In addition, once the subjects made the mental representation with the focus word, the contents of the sentence may have been easily accessed via the men- 
tal representation. Thus, it became easier for the subjects to read and comprehend the sentences by using these mental representations, making the effects of sentence length relatively equivocal.

In addition, another explanation for why the subjects did not use a task-switching strategy is that the target word is not the last word of the sentence, as it was in Towse et al. $(1998,2000)$. When the target word is the last word of the sentence, it is easier for subjects to switch to maintaining the word after they finish reading the sentence. With the present RST, however, the subjects would have had to read back to the target word if they were using a task-switching strategy, and this is highly unlikely, given the short intersentence interval. Thus, the present results suggest that subjects do not always depend on a task-switching strategy with RSTs.

Individual differences in focus effect. The results of the comparison of span scores between high-span subjects and low-span subjects revealed a significant effect of focus for low-span subjects, but not for high-span subjects. These results showed that low-span subjects had a severely impaired ability to maintain the target word with the nonfocused RST.

We also confirmed the focus effect on intrusion errors. Moreover, intrusion errors increased more for low-span subjects than for high-span subjects. These results support other studies that have indicated that low-span subjects have a deficit in inhibiting irrelevant information with the task (May et al., 1999).

In the present experiments, however, the number of focusintrusions was significantly larger than that of nonfocus intrusion, and this difference was found only in low-span subjects, and not in high-span subjects. The results suggest that low-span subjects have more difficulty inhibiting information, especially the focus word, than do high-span subjects. If low-span subjects had difficulty inhibiting any kind of target word, they would have shown a worse performance even with the focused RST, similar to that with the nonfocused RST. In fact, intrusion errors were not so frequent with the focused RST. Even low-span subjects can manage to inhibit irrelevant information with the focused RST. Thus, it was not that the low-span subjects could not inhibit irrelevant information but only that they could not inhibit irrelevant information after they had focused on it.

\section{GENERAL DISCUSSION}

The results of both experiments confirmed the focus effect. Furthermore, the focus effect was more dominant for low-span subjects than for high-span subjects in Experiment 2. Several arguments are possible, on the basis of our data, that would explain the differences in focus effect between high-span subjects and low-span subjects.

One is that low-span subjects have difficulty establishing a mental focus. In most research in which the focus effect was explored, the focus was defined by the preceding question (Blutner \& Sommer, 1988; Cutler \& Fodor, 1979) or by controlling the sentence syntactically (Birch \& Garnsey, 1995). In the present research, the focus word was not preceded by a question, nor was it stressed syntactically. Therefore, semantic processing was required for the subjects to find a focus word mentally. Establishing a mental focus requires information about word meaning, along with syntactic, pragmatic, and semantic elaboration related to sentence processing. According to Cutler and Fodor, an active search for an accented word can be interpreted as a search for sentence focus. For the subjects in the present experiments, an active search for sentence focus was, likewise, required. Once the subjects had identified the focus word in the sentence, this pivot word automatically accelerated sentence comprehension processes.

It is plausible to suggest that such an active search for a focus word - that is, establishing a mental focus - can be accomplished more efficiently by high-span subjects. This would be in agreement with Just and Carpenter (1992), who concluded that semantic or syntactic information must be activated efficiently in high-span subjects. In addition, various information must be drawn from long-term memory (Baddeley, 2000; Ericsson \& Kintsch, 1995) and must also be extracted from domain general resources (Engle, Kane, \& Tuholski, 1999) to establish a mental focus, all of which may contribute to the difficulty for low-span subjects. When these points are taken into consideration, one may conclude that low-span subjects may have difficulty in an active search for a focus word during reading and that a delay in identifying the focus word may cause a delay or an interruption in comprehension processing.

Cutler and Fodor (1979) suggested that the subjects in their phoneme-monitoring experiments were able to perform a divided attention task, listening for the target and comprehending the sentence simultaneously. Completion of the focus-identifying task gave the subjects greater processing capacity for other tasks. They argued that sentence understanding is facilitated by rapid identification of focused information. Thus, readers who quickly identify and effectively establish the mental focus for temporal integration can obtain high performance with an RST. Thus, one explanation for the differences between high-span subjects and low-span subjects is that there is a lack of facilitation in the comprehension processes, owing to a deficit in establishing mental focus.

Another argument is that low-span subjects have a deficit in inhibiting or updating their mental focus. Instead of sentences, De Beni et al. (1998) used random strings of words and asked subjects to recall the last word of each sequence while tapping a hand when an animal noun appeared in the word string. They measured the intrusion errors for the target words the subjects recalled. Their results showed that poor comprehenders made more intrusion errors than did good comprehenders. Moreover, for the intrusion errors made, intrusions more frequently involved animal nouns than other nontarget words. The results suggested that the low comprehenders had difficulty inhibiting words that required other forms of attention.

The present results coincided with the previous findings in De Beni et al. (1998). The low-span subjects had difficulty inhibiting words after they had paid attention to them. One could speculate that the updating or reset mech- 
anisms used to inhibit the focus word did not function well for them once they had built a mental focus.

In Gernsbacher and Faust (1991), it was reported that for less skilled readers, an ignored stimulus was still activated even as long as $1 \mathrm{sec}$ later. In accord with these findings, we propose that the low-span subjects in our study took a longer time to update the mental focus than did the high-span subjects and that this deficit delay affected inhibition of focus.

The RST is assumed to measure the contents of working memory similar to the central executive (Baddeley, 1992; Just \& Carpenter, 1992). In addition, the central executive is responsible for the allocation and coordination of the brain's attentional resources (Baddeley, 1996; Cowan, 1999; Engle et al., 1999). Therefore, inhibiting and updating mental focus is involved in the attentional control system of the central executive. A weak attention control system may account for the low-span subjects' having difficulty inhibiting and updating mental focus and, on the contrary, high-span subjects' having flexible attentional control systems that facilitate the updating and inhibiting of their mental focus.

One last argument is that the conflict between the focus word and the target word contributed to the performance difference between the high-span subjects and the lowspan subjects. With the nonfocused RST, both the focus word and the target word should have been activated. This being the case, the subjects would then have had to decide which one was relevant to the goals of the task-that is, the target word. Nonetheless, the focus word may have interfered with the target word, since it had already been activated during reading. This conflict between the two words is similar to the conflict in the Stroop task (Stroop, 1935).

It is likely that low-span subjects are strongly affected by conflict. When two kinds of information are activated, low-span subjects have difficulty selecting the appropriate one. Thus, there was a deficit for the low-span subjects in this experiment, not because they had trouble inhibiting irrelevant information, but because they had a deficit in inhibiting irrelevant information when they faced a conflict situation. It is also proposed that they might have had a deficit in monitoring the situation; confronted with this conflict, they may have lost track of what was required or what the goals of the task were.

A recent fMRI study indicated that the neural mechanism of the attention control system in working memory is divided into separate systems, one responsible for maintaining attention control and another for conflict monitoring (Smith \& Jonides, 1999). By using the Stroop task, the neural bases of attention control, particularly conflict monitoring, were measured (Bush, Luu, \& Posner, 2000; MacDonald, Cohen, Stenger, \& Carter, 2000). It was shown that a conflict situation mostly activated the anterior cingulate cortex (ACC), whereas attention maintenance activation was shown in the dorsolateral prefrontal cortex (DLPFC). These findings suggest that, during a conflict situation, there is a dissociation of the attention control system into attention maintenance and monitoring. With respect to the above assertion, the poor performance of the low- span subjects would have been caused by a deficit of the monitoring system in the conflict situation.

Using a listening span task, Osaka et al. (2001) measured the neural bases of differences between high-span subjects and low-span subjects. They found activity differences located only in the ACC, and not in the DLPFC, which suggests that activity in this area of the attention control system is particularly related to inhibition mechanisms associated with the monitoring system.

When faced with a potentially confusing task, such as with the nonfocused RST, low-span subjects have a deficit in controlling attention, disabling their ability to keep track of the goals of the task or of which word they should maintain. Another possibility is that low-span subjects hardly understand the situation when confronted with such a conflict; having a weaker monitoring system, they do not recognize the conflict and, hence, do not respond with a strategy for handling the task.

It appears difficult to determine which explanation is the best, and further investigation will be needed. Here, we consider that low-span subjects have difficulty with a variety of processes. A delay in establishing a focus word will precipitate a disturbance in later inhibitory responses, or vice versa.

The present results suggest that differences between high- and low-span subjects are related to the efficiency of establishing a focus and inhibiting and updating that focus in conflict situations. The important factor influencing the performance of subjects on the RST is how well they control attention while establishing a mental focus and then, subsequently, allocating this attention for updating the mental focus and inhibiting the irrelevant information. Moreover, theories in which the role of attention allocation with the RST is implicated, such as the one presented above, by accounting for higher order cognition, easily lend themselves to explanations of other span tasks, such as the operation span task or the counting span task (Engle et al., 1999; Turner \& Engle, 1989). Both of these other kinds of span tasks also require the attention controller for the dual task.

On the basis of the results, the factors that contribute to individualdifferences with the RST are related to the mental faculties of establishing and inhibiting mental focus in reading. We conclude that the focus effect is a reflection of the general properties of working memory.

\section{REFERENCES}

Amano, N., \& Kondo, K. (1999). Lexical properties of Japanese. Tokyo: Sanseido.

BADDElEy, A. D. (1986). Working memory. New York: Oxford University Press.

BAdDEley, A. D. (1992). Working memory. Science, 255, 556-559.

BADDELEY, A. D. (1996). Exploring the central executive. Quarterly Journal of Experimental Psychology, 49A, 5-28.

BADDELEY,A. D. (2000). The episodic buffer: A new component of working memory? Trends in Cognitive Sciences, 4, 417-423.

Baddeley, A. D., Logie, R., Nimmo-Smith, I., \& Brereton, R. (1985). Components of fluent reading. Journal of Memory \& Language, 24, 119-131.

Birch, S. L., \& GARnSEy, S. M. (1995). The effect of focus on memory for words in sentence. Journal of Memory \& Language, 34, 232-267. 
Blutner, R., \& Sommer, R. (1988). Sentence processing and lexical access: The influence of the focus-identifying task. Journal of Memory \& Language, 27, 359-367.

Bolinger, D. (1986). Intonation and its parts. Stanford, CA: Stanford University Press.

Bush, G., LuU, P., \& Posner, M. I. (2000). Cognitive and emotional influences in anterior cingulate cortex. Trends in Cognitive Sciences, $\mathbf{4}$ 215-222.

Carpenter, P. A., \& Just, M. A. (1977). Integrative processes in comprehension. In P. LaBerge \& S. J. Samuels (Eds.), Basic processes in reading: Perception and comprehension (pp. 217-241). Hillsdale, NJ: Erlbaum.

Carpenter, P. A., \& Just, M. A. (1989). The role of working memory in language comprehension. In D. Klar \& K. Kotovsky (Eds.), Complex information processing: The impact of Herbert A. Simon (pp. 3168). Hillsdale, NJ: Erlbaum.

Chомsкy, N. (1971). Deep structure, surface structure and semantic interpretation. In D. D. Steinberg \& L. A. Jakobovits (Eds.), Semantics: An interdisciplinary reader in philosophy, linguistics, and psychology (pp. 183-216). New York: Cambridge University Press.

Conway, A. R. A., \& ENgle, R. W. (1994). Working memory and retrieval: A resource-dependent inhibition model. Journal of Experimental Psychology: General, 123, 354-373.

CowAN, N. (1999). An embedded-processes model of working memory. In A. Miyake \& P. Shah (Eds.), Models of working memory: Mechanisms of active maintenance and executive control (pp. 62-101). New York: Cambridge University Press.

CUtLER, A. (1976). Phoneme-monitoring reaction time as a function of preceding intonation contour. Perception \& Psychophysics, 20, 55-60.

Cutler, A., \& Fodor, J. A. (1979). Semantic focus and sentence comprehension. Cognition, 7, 49-59.

DANEMAN, M., \& CARPENTER, P. A. (1980). Individualdifferences in working memory and reading. Journal of Verbal Learning \& Verbal Behavior, 19, 450-466.

Daneman, M., \& Carpenter, P. A. (1983). Individual differences in integrating information between and within sentences. Journal of Experimental Psychology: Learning, Memory, \& Cognition, 9, 561-583.

DANEMAN, M., \& MeriKLe,P. M. (1996). Working memory and language comprehension: A meta-analysis. Psychonomic Bulletin \& Review, $\mathbf{3}$, 422-433.

De Beni, R., Palladino, P., Pazzaglia, F., \& Cornoldi, C. (1998). Increases in intrusion errors and working memory deficit of poor comprehenders. Quarterly Journal of Experimental Psychology, 51A, 305-320.

Engle, R.W., Conway, A. R. A., Tuholski, S. W., \& Shisler, R J. (1995) A resource account of inhibition. Psychological Science, 6, 122-125.

Engle, R.W., Kane, M. J., \& TuholsKi, S. W. (1999). Individual differences in working memory capacity and what they tell us about controlled attention, general fluid intelligence, and functions of the prefrontal cortex. In A. Miyake \& P. Shah (Eds.), Models of working memory: Mechanisms of active maintenance and executive control (pp. 102-134). New York: Cambridge University Press.

ERICSSON, K. A., \& KINTSCH, W. (1995). Long-term working memory. Psychological Review, 102, 211-245.

Gernsbacher, M. A. (1990). Less skilled readers have less efficient suppression mechanisms. Psychological Science, 4, 294-298.

Gernsbacher, M. A., \& FAust, M. E. (1991). The mechanism of suppression: A component of general comprehension skill. Journal of Experimental Psychology: Learning, Memory, \& Cognition, 17, 245-262.

HallidaY, M. A. K. (1967). Notes on transitivity and theme in English: Pt. 2. Journal of Linguistics, 3, 199-244.

Hornby, P. A. (1974). Surface structure and presupposition. Journal of Verbal Learning \& Verbal Behavior, 13, 530-538.

JACKENDOFF, R. (1972). Semantic interpretation in generative grammar. Cambridge, MA: MIT Press.

Just, M. A., \& CARPEnTER, P. A. (1992). A capacity theory of comprehension: Individual differences in working memory. Psychological Review, 99, 122-149.

KinTSCH, W., \& VAN DisK, T. A. (1978). Toward a model of context comprehension and production. Psychological Review, 85, 363-394.
KoIzumI, T. (1978). Japanese orthography. Tokyo: Taisyukan. [In Japanese]

KunO, S. (1980). Discourse and grammar. Tokyo: Taishukan-syoten. [In Japanese]

La Pointe, L. B., \& EngLe, R. W. (1990). Simple and complex word spans as measures of working memory capacity. Journal of Experimental Psychology: Learning, Memory, \& Cognition, 16, 1118-1133.

MacDonald, A. W., III, Cohen, J. D., Stenger, V. A., \& Carter, C. S. (2000). Dissociating the role of the dorsolateral prefrontal and anterior cingulated cortex in cognitive control. Science, 288, 1835-1838.

Masson, M. E. J., \& Miller, J. A. (1983). Working memory and individual differences in comprehension and memory of text. Journal of Educational Psychology, 75, 314-318.

May, C. P., HASHER, L., \& KAne, M. J. (1999). The role of interference in memory span. Memory \& Cognition, 27, 759-767.

OsAKa, M., \& OsAKA, N. (1992). Language-independent working memory as measured by Japanese and English reading span tests. Bulletin of the Psychonomic Society, 30, 287-289.

OSAKA, M., \& OSAKA, N. (1994). Working memory capacity related to reading: Measurement with the Japanese version of reading span test. Japanese Journal of Psychology, 65, 339-345. [In Japanese with English summary]

Osaka, M., Osaka, N., Kondo, H., Morishita, M., Fukuyama, H., Aso, T., \& SHIBASAKI, H. (2001). Individual differences in verbal working memory: An fMRI study. Clinical Neurophysiology, 112, 1123.

Perfetti, C. A., \& Goldman, S. R. (1976). Discourse memory and reading comprehension skill. Journal of Verbal Learning \& Verbal Behavior, 15, 33-42.

Smith, E. E., \& Jonides, J. (1999). Storage and executive processes in the frontal lobes. Science, 283, 1657-1661.

Stroop, J. R. (1935). Studies of interference in serial verbal reactions. Journal of Experimental Psychology, 18, 643-662.

Towse, J. N., Hiтch, G. J., \& HutTon, U. (1998). A reevaluation of working memory capacity on children. Journal of Memory \& Language, 39, 195-217.

Towse, J. N., Hitch, G. J., \& Hutton, U. (2000). On the interpretation of working memory span in adults. Memory \& Cognition, 28, 341-348.

Turner, M. L., \& ENGLE, R. W. (1989). Is working memory capacity task dependent? Journal of Memory \& Language, 28, 127-154.

\section{NOTES}

1. We calculated the total number of words correctly recalled from all the trials in Experiment 1. The mean number of words correctly recalled for the focused and nonfocused RSTs was $57.70(S D=7.04)$ and 50.37 $(S D=9.99)$, respectively.

2. Standard versions of the Japanese RST (Osaka \& Osaka, 1992, 1994) did not control for whether or not the target word was the focus word of the sentence. Although about half of the target words across the RST were the most important word for comprehending the sentence, their evaluation values were not necessarily above $70 \%$.

3 . We calculated the total number of words correctly recalled from all the trials in Experiment 2. The mean number of words correctly recalled by high-span subjects during the focused RST task was $37.52(S D=$ $3.82)$ in the short-sentence condition and $38.52(S D=2.9)$ in the longsentence condition. During the nonfocused RST task, the number of correctly recalled words was $36.40(S D=4.36)$ in the short-sentence condition and $37.30(S D=3.36)$ in the long-sentence condition.

For low-span subjects, the mean number of words correctly recalled during the focused RST task was $29.82(S D=5.28)$ in the short-sentence condition and $31.74(S D=5.60)$ in the long-sentence condition. During the nonfocused RST task, the number of correctly recalled words was $27.08(S D=7.11)$ in the short-sentence condition and $27.08(S D=$ $6.09)$ in the long-sentence condition.

(Manuscript received June 19, 2001; revision accepted for publication February 14, 2002.) 